\title{
Malignant syphilis: \\ a clinical form of syphilis to consider
}

Mercè Alsina-Gibert, Daniel Morgado-Carrasco, Xavier Fustà-Novell, Alicia Barreiro Capurro, Irene Fuertes De Vega

\section{Introduction and Objectives}

Malignant syphilis is an uncommon nodular-ulcerative form of secondary syphilis that was first described in 1859. It has been considered extremely rare since the beginning of the antibiotic era. However, in the last two decades there has been a resurgence of this unusual form of syphilis, mainly attributed to HIV co-infection in young and middle-aged men. Our objective was to review all diagnosed cases of malignant syphilis at our centre in the last five years and define its clinical and laboratory characteristics.

\section{Results}

Of the 332 patients diagnosed with syphilis 4 had MS (1.2\%). All patients (4/4) were men who have sex with men, HIV+ (between 26 and 54 years-old), and had positive treponemal and non-treponemal tests.

$3 / 4$ were not receiving treatment for HIV at the time of diagnosis of syphilis, with the infection being unknown in one of them

4/4 presented multiple ulcerated nodules, palmoplantar involvement and 3/4 had affectation of the scalp.

One patient presented with neurosyphilis. CD4+ cell counts were $171 / \mathrm{mm}^{3}, 250 / \mathrm{mm}^{3}$, $697 / \mathrm{mm}^{3}$ and $790 / \mathrm{mm}^{3}$.

The two patients with a lower CD4+ count and prozone phenomenon in the serology developed a Jarisch-Herxheimer reaction when starting the antibiotic treatment.

$3 / 4$ were treated with three weekly intramuscular doses of 2.4 million units of benzathine penicillin $\mathrm{G}$. Intravenous penicillin $\mathrm{G}$ was administered intravenously during 15 days to the patient with neurosyphilis.

Complete clinical response was observed in all patients one month after therapy.

\section{Materials and Methods}

A review of medical records from all patients diagnosed of syphilis in a tertiary referral hospital from January 2012 to December 2016 was done. Patients diagnosed as malignant or nodular-ulcerative syphilis were recruited. A retrospective review of medical records, clinical photographs, histological findings, laboratory analysis, treatments, and outcomes was carried out.

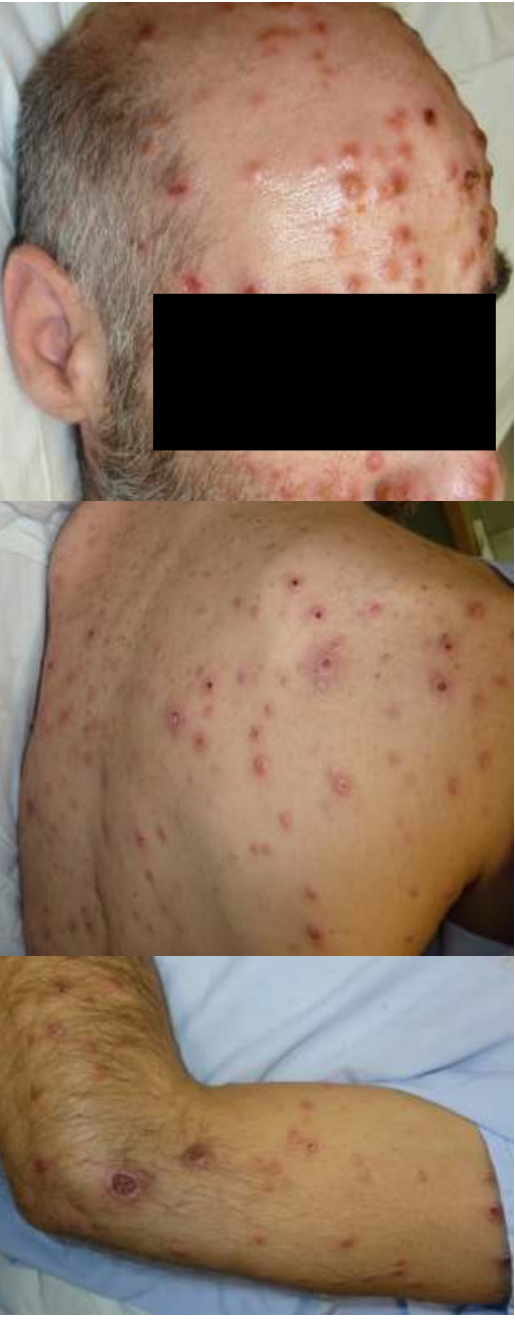

Patient 1

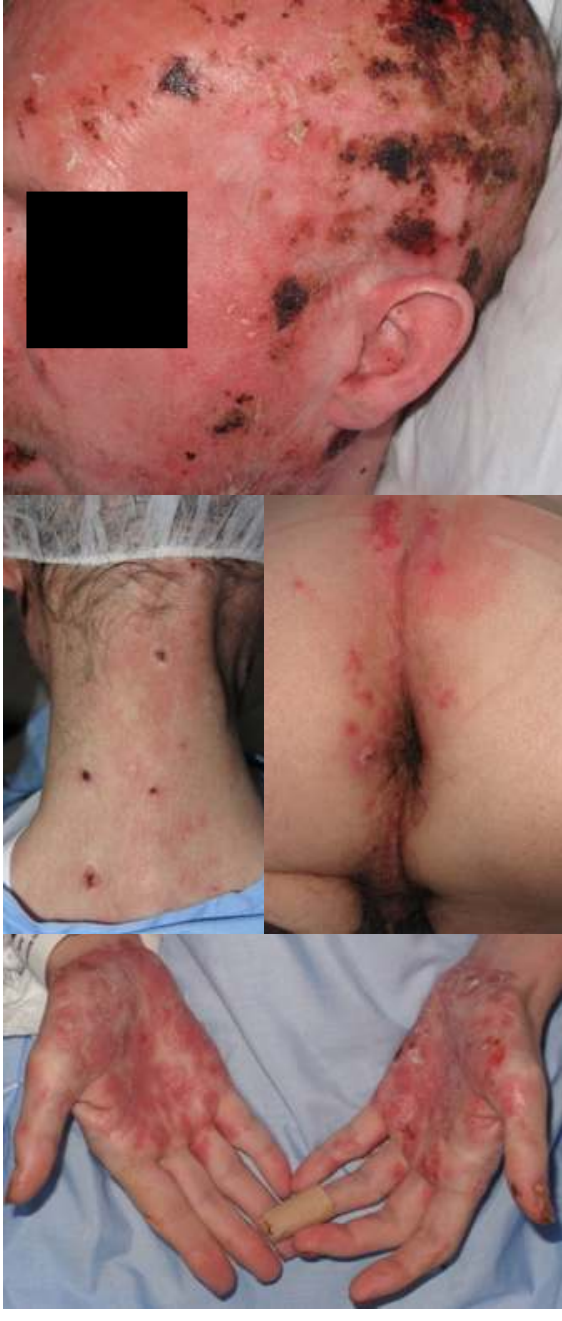

Patient 2

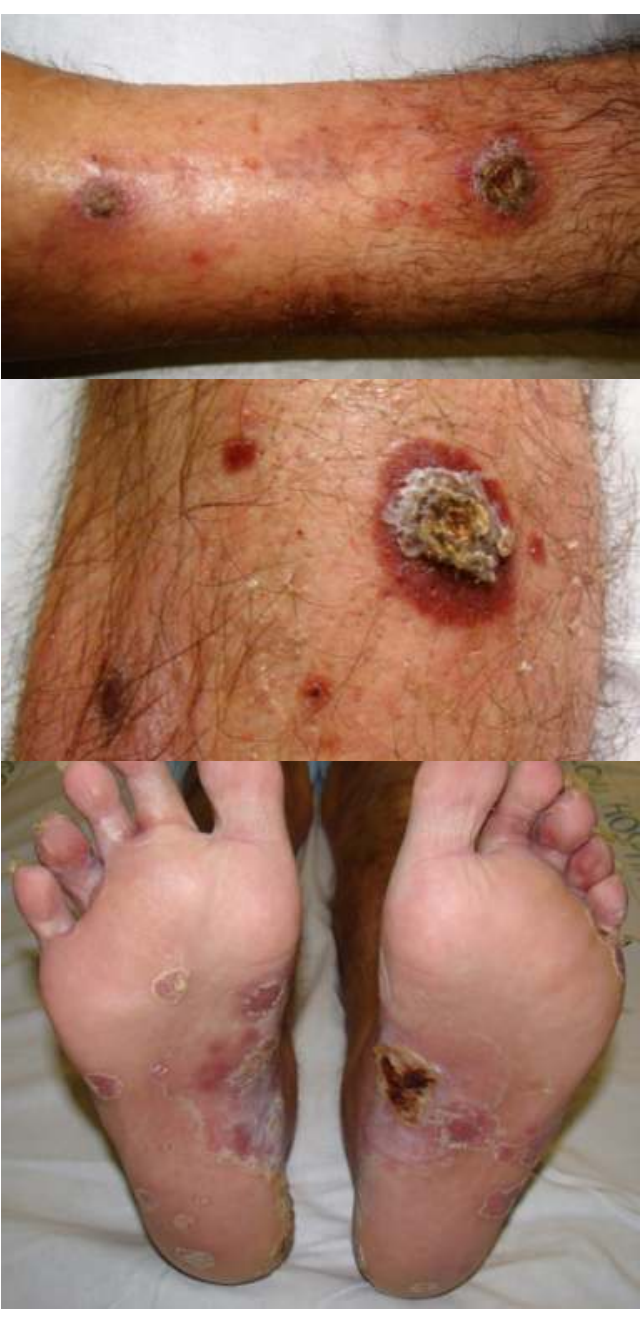

Patient 3

Table I. Patients' characteristics

\begin{tabular}{|c|c|c|c|c|c|c|c|c|c|c|c|}
\hline $\begin{array}{l}\text { Case } \\
\text { number }\end{array}$ & Sex & Age & $\begin{array}{l}\text { Mucocutaneous } \\
\text { manifestations }\end{array}$ & $\begin{array}{l}\text { Systemic } \\
\text { symptoms }\end{array}$ & HIV & $\begin{array}{l}\text { Viral charge HIV } \\
\text { (copies/ml) }\end{array}$ & TCD4+ (cells $\left./ \mathrm{mm}^{3}\right)$ & Histological findings & VDRL & Treatment & Outcome \\
\hline Case 1 & $\begin{array}{c}M \\
(M S M)\end{array}$ & 39 & $\begin{array}{c}\text { Ulcerated and necrotic nodules } \\
\text { Involvement of palms and soles } \\
\text { Scalp lesions }\end{array}$ & Fever $\left(>38^{\circ} \mathrm{C}\right)$ & $\begin{array}{c}+ \\
\text { (no treatment) }\end{array}$ & 24,950 & 171 & $\begin{array}{l}\text { Lichenoid dermatitis } \\
\text { Treponemal IHC (+) }\end{array}$ & $\begin{array}{c}1 / 128 \\
\text { Prozone } \\
\text { phenomenon }\end{array}$ & $\begin{array}{l}\text { Intramuscular } \\
\text { penicillin } G \\
\text { benzathine } \\
2,400,000 \text { units }(x 3)\end{array}$ & $\begin{array}{l}\text { Jarisch- } \\
\text { Herxheimer } \\
\text { reaction. } \\
\text { Complete clinical } \\
\text { response }\end{array}$ \\
\hline Case 2 & $\begin{array}{c}\mathrm{M} \\
(\mathrm{MSM})\end{array}$ & 36 & $\begin{array}{l}\text { Ulcerated and necrotic nodules } \\
\text { Involvement of palms and soles } \\
\text { Scalp and nail lesions } \\
\text { Condiloma lata }\end{array}$ & $\begin{array}{l}\text { Fever }\left(>38^{\circ} \mathrm{C}\right) \\
\text { Syphilitic uveitis } \\
\text { Neurosyphilis }\end{array}$ & $\begin{array}{c}\stackrel{+}{\text { (no treatment) }} \\
\text { (n) }\end{array}$ & $1,350,000$ & 250 & $\begin{array}{c}\text { Spongiotic dermatitis } \\
\text { Treponemal IHC (+) }\end{array}$ & $\begin{array}{c}1 / 250 \\
\text { Prozone } \\
\text { phenomenon }\end{array}$ & $\begin{array}{c}\text { Intravenous } \\
\text { aqueous crystalline } \\
\text { penicillin } \mathrm{G} \\
24,000,000 \text { units } \\
\text { daily for } 15 \text { days }\end{array}$ & $\begin{array}{l}\text { Jarisch- } \\
\text { Herxheimer } \\
\text { reaction. } \\
\text { Complete clinical } \\
\text { response }\end{array}$ \\
\hline Case 3 & $\begin{array}{c}M \\
(M S M)\end{array}$ & 54 & $\begin{array}{l}\text { Ulcerated and necrotic nodules } \\
\text { Involvement of palms and soles }\end{array}$ & Fever (>38으) & $\stackrel{+}{\stackrel{+}{\text { (no treatment) }}}$ & 71,300 & 697 & $\begin{array}{l}\text { Lichenoid dermatitis } \\
\text { Treponemal IHC (-) }\end{array}$ & $1 / 512$ & $\begin{array}{c}\text { Intramuscular } \\
\text { penicillin } G \\
\text { benzathine } \\
2,400,000 \text { units }(x 3)\end{array}$ & $\begin{array}{c}\text { Complete clinical } \\
\text { response }\end{array}$ \\
\hline Case 4 & $\begin{array}{c}M \\
(M S M)\end{array}$ & 26 & $\begin{array}{c}\text { Ulcerated and necrotic nodules } \\
\text { Involvement of palms and soles } \\
\text { Scalp lesions }\end{array}$ & Fever & $\stackrel{+}{+}$ & $<37$ & 790 & $\begin{array}{c}\text { Areas of } \\
\text { abscessification } \\
\text { Treponemal IHC (-) }\end{array}$ & $1 / 256$ & $\begin{array}{c}\text { Intramuscular } \\
\text { penicillin } G \\
\text { benzathine } \\
2,400,000 \text { units }(x 1)\end{array}$ & $\begin{array}{l}\text { Complete clinical } \\
\text { response }\end{array}$ \\
\hline
\end{tabular}

Abbreviations: $M$, male; MSM, men sex with men; IHC, immunohistochemistr

\section{Conclusions}

The increasing incidence of syphilis among HIV+ patients has lead to the resurgence of malignant syphilis. This form of syphilis is almost always associated with HIV co-infection. However, it seems that severe immunosuppression is not a mandatory requirement for its occurrence.

Neurosyphilis, a prozone phenomenon, and a Jarisch-Herxheimer reaction are frequently associated findings. Real incidence of malignant syphilis among HIV-positive patients and its possible complications are still unknown.

\section{References}

1. Cid PM, Cudós ES, Zamora Vargas FX, Merino MJB, Pinto PH. Pathologically confirmed malignant syphilis using immunohistochemical staining: report of 3 cases and review of the literature. Sex Transm Dis. 2014;41(2):94-7.

2. Witkowski JA, Parish LC. The great imitator: malignant syphilis with hepatitis. Clin Dermatol. 2002;20(2):156-63.

3. Kumar B, Muralidhar S. Malignant syphilis: a review. AIDS Patient Care STDS. 1998;12(12):921-5.

4. Don PC, Rubinstein R, Christie S. Malignant syphilis (lues maligna) and concurrent infection with HIV. Int J Dermatol. 1995;34(6):403-7. 